\title{
Predischarge Prediction of Readmission After Cytoreductive Surgery and Hyperthermic Intraperitoneal Chemotherapy: Derivation and Validation of a Risk Prediction Score
}

\author{
Caroline J. Rieser, MD ${ }^{1}$ (D), Lauren B. Hall, MSc ${ }^{1}$, Eliza Kang, BS ${ }^{1}$, Amer H. Zureikat, MD ${ }^{1}$, \\ Matthew P. Holtzman, MD ${ }^{1}$, James F. Pingpank, MD ${ }^{1}$, David L. Bartlett, MD $^{2}$, and M. Haroon A. Choudry, MD ${ }^{1}$ \\ ${ }^{1}$ Division of Surgical Oncology, Koch Regional Perfusion Center, University of Pittsburgh, Pittsburgh, PA; ${ }^{2}$ AHN Cancer \\ Institute, Allegheny Health Network, Pittsburgh, PA
}

\begin{abstract}
Background. Ninety-day hospital readmission rates following cytoreductive surgery and hyperthermic intraperitoneal chemotherapy (CRS/HIPEC) range from 20 to $40 \%$.

Objective. The aim of this study was to develop and validate a simple score to predict readmissions following CRS/HIPEC.

Study Design. Using a prospectively maintained database, we retrospectively reviewed clinicopathologic, perioperative, and day-of-discharge data for patients undergoing CRS/HIPEC for peritoneal surface malignancies between 2010 and 2018. In-hospital mortalities and discharges to hospice were excluded. Multivariate logistic regression was utilized to identify predictors of unplanned readmission, with three-quarters of the sample randomly selected as the derivation cohort and one-quarter as the validation cohort. Using regression coefficient-based scoring methods, we developed a weighted 7-factor, 10-point predictive score for risk of readmission.

Results. Overall, 1068 eligible discharges were analyzed; 379 patients were readmitted within 90 days (35.5\%).
\end{abstract}

Supplementary Information The online version of this article $(\mathrm{h}$ ttps://doi.org/10.1245/s10434-020-09547-7) contains supplementary material, which is available to authorized users.

(C) The Author(s) 2021

First Received: 29 July 2020

Accepted: 13 December 2020;

Published Online: 23 January 2021

C. J. Rieser, MD

e-mail: riesercj2@upmc.edu
Seven factors were associated with readmission: stoma creation, Peritoneal Cancer Index score $\geq 15$, hyponatremia, in-hospital major complication, preoperative chemotherapy, anemia, and discharge to nursing home. In the validation cohort, 25 patients $(9.2 \%)$ were categorized as high risk for readmission, with a predicted rate of readmission of $69.3 \%$ and an observed rate of $76.0 \%$. The score had fair discrimination (area under the curve 0.70) and good calibration (Hosmer-Lemeshow goodness-of-fit $p$-value of 0.77).

Conclusion. Our proposed risk score, easily obtainable on day of discharge, distinguishes patients at high risk for readmission over 90 days following CRS/HIPEC. This score has the potential to target high-risk individuals for intensive follow-up and other interventions.

Cytoreductive surgery and hyperthermic intraperitoneal chemotherapy (CRS/HIPEC) is an aggressive and complex treatment for patients with disseminated peritoneal malignancies. Due to the high rates of morbidity, postoperative care is often as complex as the surgery itself. ${ }^{1,2}$ As a consequence, readmissions following CRS/HIPEC are frequent, occurring after $20-40 \%$ of discharges. ${ }^{1,3}$ With increasing regionalization and fragmentation of surgical care, up to $20 \%$ of surgical oncology readmissions may occur at non-index hospitals, with associated increased risk for major complications. ${ }^{3-5}$ In addition to impacting patient quality of life and cost of care, readmissions and associated complications may both drive worse cancer-specific survival. ${ }^{1,4,6}$

While many interventions are currently being proposed to reduce readmissions following complex oncologic surgery, there is no validated tool for predicting readmission 
for patients undergoing CRS/HIPEC to guide application and track outcomes for such interventions. Current surgeryspecific risk prediction tools heavily weight complications and cancer diagnoses, rendering them inappropriate for use in the CRS/HIPEC population, where all patients have disseminated cancer and upwards of $60 \%$ of patients experience at least one postoperative complication and $26 \%$ experience a major complication. ${ }^{1,2,7,8}$

To efficiently target patients for interventions aimed at decreasing readmission, we need to first reliably identify a high-risk population prior to discharge. This would allow providers to appropriately direct future efforts aimed at improving patient care and oncologic outcomes. The aim of this study was to examine the drivers of readmission among patients undergoing CRS/HIPEC and derive and validate a simple score to predict the risk of readmission at discharge.

\section{METHODS}

\section{Study Design and Population}

A retrospective cohort study was conducted of all patients undergoing CRS/HIPEC at a single-center, quarternary surgical oncology hospital that is part of a 40-hospital healthcare system, from 1 January 2010 through 31 December 2018, using a prospectively maintained database. Patients were excluded from the analysis if they died during, or were discharged to hospice after, index hospitalization. Planned readmissions were excluded from this analysis. This study was approved by the Institutional Review Board at the University of Pittsburgh (IRB 19010278).

\section{Study Outcome}

Our study outcome of interest was readmission following hospitalization for CRS/HIPEC. We defined readmission as unplanned hospitalization of any duration within 90 days of discharge. ${ }^{9}$ These included readmissions to both index and non-index facilities. Admissions to skilled nursing facilities or long-term acute care facilities following discharge were not counted as readmissions. ${ }^{10}$

A secondary outcome of interest was readmission due to surgical quality of care. This was chosen as this subset of readmissions would be theoretically targetable for perioperative interventions to prevent readmissions. Readmissions were labeled as related to surgical quality of care based on the presence of International Classification of Diseases (ICD) codes for readmission diagnoses established by Mull and colleagues ${ }^{11}$ via a Delphi consensus process. Readmissions with diagnoses of infection, sepsis, pneumonia, hemorrhage/hematoma, anemia, ostomy complications, acute renal failure, failure to thrive, fluid/electrolyte disorders, or venous thromboembolism (VTE) were considered to be associated with surgical quality. Patients could have more than one cause for readmission if more than one relevant diagnosis code was present on readmission.

\section{Predictor Variables}

Data were collected from several sources for this analysis, including preoperative evaluation, perioperative record, postoperative course, and day-of-discharge characteristics. The following patient characteristics were examined: sex, body mass index (BMI, $\mathrm{kg} / \mathrm{m}^{2}$ ), age-adjusted Charlson-Deyo Comorbidity Index (AA-CCI), smoking status, repeat CRS/HIPEC, and American Society of Anesthesiologists' (ASA) physical status score. In addition, the following oncologic factors were also examined: primary histology, Eastern Cooperative Oncology Group (ECOG) score, preoperative chemotherapy receipt, and number of preoperative chemotherapy cycles.

The following operative details were examined: prior surgery score (PSS; $0=$ no surgery or biopsy only; 1 = exploratory laparotomy, only 1 region dissected; $2=$ exploratory laparotomy with resections, two to five regions dissected; and $3=$ extensive previous cytoreduction, more than five regions dissected), Peritoneal Cancer Index (PCI) score as determined at the time of operation, operative length (hours), estimated blood loss (EBL, mL), intraoperative transfusion, number of visceral resections, number of anastomoses, and completeness of cytoreduction (CC) score.

The following hospitalization details were collected: length of stay (days), in-hospital complication (any), comprehensive complication index score (CCI total), major (Clavien-Dindo grade III or higher) complication rate, inhospital anastomotic leak, in-hospital VTE event, postoperative transfusion, percutaneous drain placement, inhospital need for total parenteral nutrition (TPN), return to the intensive care unit (ICU) during index admission, and return to the operating room (OR) during index admission.

Several discharge factors were examined: discharge with stoma (ileostomy or colostomy), day-of-discharge hemoglobin $(\mathrm{g} / \mathrm{dL})$, white blood cell count $\left(10^{9}\right.$ cells/L) and sodium $(\mathrm{mEq} / \mathrm{L})$ levels, discharge on antibiotics, therapeutic anticoagulation, TPN, number of discharge medications, and discharge to a skilled nursing facility

All variables were chosen a priori based on previously published literature. . $^{3,7,8,10,12-14}$ 


\section{Missing Data}

Missingness was quantified for all predictor variables (electronic supplementary Table 1), was minimal, and was $<5 \%$, with the exception of the number of pre-CRS/HIPEC chemotherapy cycles $(28 \%)$. We used a multiple imputation approach using 10 imputed datasets via chained multiple imputations to handle missing data. ${ }^{15}$

\section{Statistical Methods}

The cohort was randomized into a derivation (75\%) set and a separate validation (25\%) set. Descriptive statistics were reported for the whole cohort and the derivation cohort by readmission status. Continuous data were reported as mean and standard deviation (SD) when normally distributed, and median with interquartile range (IQR) when non-normally distributed. Categorical data were reported as frequencies and percentages.

All perioperative variables were evaluated for possible significance in predicting readmission via univariate logistic regression in the derivation cohort. Variables with a $p$-value $<0.30$ on univariate analysis were included in an initial backwards stepwise elimination multivariable logistic regression model. Using backwards elimination, variables were sequentially removed from the model until all remaining predictors had a $p$-value $<0.05 .{ }^{10}$ For ease of interpretation and application into a simple score, in the final model continuous variables were converted into dichotomous variables, with cut-offs determined by a minimum $p$-value approach. ${ }^{16}$ Using regression coefficientbased scoring methods, the multivariable model was then converted into an integer-based score. ${ }^{17}$

The probability of readmission was then examined by risk score within the derivation cohort and patients were categorized into groups based on predicted risk for readmission (low, moderate, and high), establishing score cutoffs for each group. ${ }^{10}$ The risk score was then applied to the validation cohort. Model calibration was assessed by examining the observed and expected rates of readmission within the validation cohort and via the Hosmer-Lemeshow goodness-of-fit test. Model discrimination was evaluated within the validation cohort by examining the receiver operating characteristic (ROC) curve and calculating the area under the ROC curve (AUC).

Model performance was evaluated between individual components and the full model by examination of the AUC. The Brier score was also used to assess overall model accuracy and performance, with possible scores ranging from 0 to 1 and lower scores indicating better performance. Spiegelhalter's z-statistic was used to test the significance of the Brier score; a $p$-value $<0.05$ indicates poor calibration.
A two-sided $p$-value $<0.05$ was considered significant for all tests. The data were analyzed using STATA 15 (StataCorp LLC, College Station, TX, USA).

\section{RESULTS}

\section{Patient Cohort}

During the study period, 1163 CRS/HIPEC procedures were performed (Fig. 1). Fifteen patients were excluded due to death during hospitalization or discharge to hospice. Eighty patients underwent planned readmission for ileostomy takedown within 90 days of discharge and were excluded from this analysis. Overall, 962 patients accounted for 1068 eligible discharges following CRS/ HIPEC. All 1068 encounters were included in the final analysis. Of these, 379 patients $(35.5 \%)$ were readmitted within 90 days, with a median time to readmission of 12 days (IQR 6-22). Of these readmissions, 288 (76.0\%) readmissions were categorized as related to quality of surgical care (Table 1). The primary reasons for readmission were infectious complications and fluid or electrolyte issues; $134(35.4 \%)$ readmissions were labeled as failure to thrive.

The 1068 encounters were randomly divided into a derivation cohort $(n=796)$ and a validation cohort $(n=272)$. Table 2 demonstrates the baseline characteristics for the overall and derivation cohorts. Median patient age was 56 years and approximately half of the cohort was male; 138 (13\%) patients underwent repeat CRS/HIPEC. The primary tumor histologies were appendiceal $(46 \%)$ and colorectal (29\%), and the majority of patients (60\%) underwent preoperative chemotherapy, with a median number of six cycles (IQR 4-10). The median PCI score was 14 (IQR 8-21).

\section{Predicting 90-Day Readmission}

Using backwards elimination, seven factors that were associated with readmission were identified in the final multivariate model $(p<0.05)$. The final predictors in the model are listed in Table 3. This model was converted to a seven-component, 10-point score based on stoma creation during operation, $\mathrm{PCI}$ score $\geq 15$, sodium level at discharge $<135 \mathrm{mEq} / \mathrm{L}$, major postoperative complications, preoperative chemotherapy, hemoglobin level on discharge $<8 \mathrm{~g} / \mathrm{dL}$, and discharge to a skilled nursing facility.

Using this score, patients were stratified into low- (0-1), moderate- (2-5), and high-risk (6+) cohorts (Fig. 2). Lowrisk patients in the derivation cohort had a predicted risk for readmission of $21.4 \%$ and an observed rate of readmission of $21.0 \%$. High-risk patients in the derivation 
FIG. 1 Study diagram. From 2010 to 2018, 1163 CRS/HIPEC procedures were performed at our institution. Fifteen patients were excluded from the analysis due to death or discharge to hospice. Among 1148 discharges, 80 had planned readmissions for secondary procedures and were excluded from the analysis, resulting in 1068 discharges being analyzed, with 379 instances $(35.5 \%)$ of readmission. $C R S$ cytoreductive surgery, HIPEC hyperthermic intraperitoneal chemotherapy

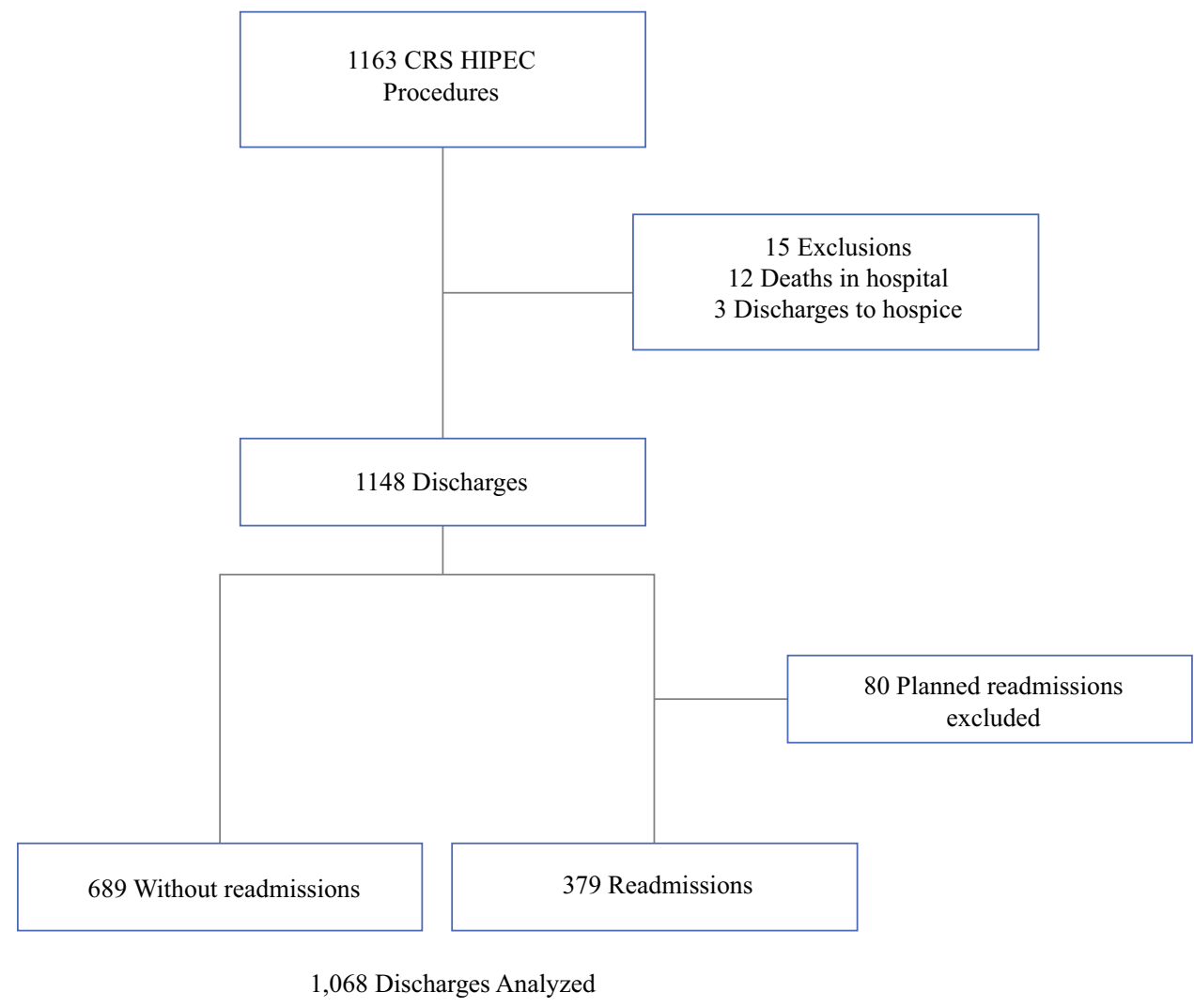

TABLE 1 Readmission details

\begin{tabular}{lc}
\hline Variable & $n(\%)$ \\
\hline 30-day readmission rate & $320(30.0)$ \\
90-day readmission rate & $379(35.5)$ \\
Non-index hospital readmission & $109(28.8)$ \\
Time to readmission, days [median (IQR)] & $12(6-22)$ \\
Admission labeled as failure to thrive & $134(35.4)$ \\
Admission related to surgical care & $288(76.0)$ \\
Infection & $162(56.3)$ \\
Fluid status or kidney function & $152(52.8)$ \\
Ostomy care or complications & $45(15.6)$ \\
Deep vein thrombosis or pulmonary embolism & $23(8.0)$ \\
\hline
\end{tabular}

cohort had a predicted risk of readmission of $69.3 \%$ and an observed rate of readmission of $79.0 \%$. The results were similar in the validation cohort (Table 4, electronic supplementary Tables 2-4).

Hosmer-Lemeshow goodness-of-fit statistics were $p=0.34$ and $p=0.77$ in the derivation and validation cohorts, respectively, indicating good calibration. The discriminatory power of the score was fair, with an AUC of 0.66 in the derivation cohort and 0.70 in the validation cohort. When applied to the whole cohort, the discrimination remained fair, with a c-statistic of 0.68 .

\section{Surgical Quality Care Readmissions}

We next examined our model performance in predicting 90-day readmissions related to quality of surgical care. In the derivation cohort, the model showed fair discrimination, with an AUC of 0.70 and good calibration with Hosmer-Lemeshow goodness-of-fit statistics $(p=0.47)$. In the validation cohort, the model had fair discrimination, with an AUC of 0.72 and good calibration with HosmerLemeshow goodness-of-fit statistics $(p=0.87)$. These suggest the model is functional not only for predicting general CRS/HIPEC readmissions but also in targeting potentially preventable, surgical quality-related readmissions.

\section{Analysis of Full Model Performance versus Individual Factors}

The discriminatory power of the full model was assessed in the validation cohort and compared with the performance of individual factors. The full model demonstrated the highest AUC (0.70) and lowest Brier score (0.200), indicating superior discrimination and calibration compared with individual elements. 
TABLE 2 Baseline characteristics and univariate analysis in the derivation cohort

\begin{tabular}{|c|c|c|c|c|}
\hline \multirow[t]{2}{*}{ Variable } & \multirow[t]{2}{*}{ Entire cohort $[n=1068]$} & \multicolumn{3}{|c|}{ Univariate analysis in the derivation set $[n=796]$} \\
\hline & & No readmission $[n=516]$ & Readmitted $[n=280]$ & $p$-Value \\
\hline \multicolumn{5}{|l|}{ Patient characteristics } \\
\hline Age, years & $56(47-64)$ & $55(47-63)$ & $57(47-65)$ & 0.22 \\
\hline Male & $525(49 \%)$ & $266(52 \%)$ & $134(48 \%)$ & 0.32 \\
\hline BMI, $\mathrm{kg} / \mathrm{m}^{2}$ & $26.9(23.7-31.2)$ & $26.8(23.6-31.0)$ & $26.9(23.9-31.4)$ & 0.32 \\
\hline AA-CCI & $6(2-8)$ & $6(2-8)$ & $4(2-8)$ & 0.09 \\
\hline Active smoking & $77(7.2 \%)$ & $52(8 \%)$ & $17(6 \%)$ & 0.25 \\
\hline Repeat CRS/HIPEC & $138(13 \%)$ & $66(13 \%)$ & $42(15 \%)$ & 0.39 \\
\hline ASA physical status & & & & 0.62 \\
\hline 1 & $1(0.1 \%)$ & $1(0.2 \%)$ & - & \\
\hline 2 & $216(20 \%)$ & $111(22 \%)$ & $55(20 \%)$ & \\
\hline 3 & $744(70 \%)$ & $352(68 \%)$ & $201(72 \%)$ & \\
\hline 4 & $107(10 \%)$ & $51(10 \%)$ & $24(8 \%)$ & \\
\hline 5 & $1(0.1 \%)$ & $1(0.2 \%)$ & - & \\
\hline \multicolumn{5}{|l|}{ Oncologic factors } \\
\hline Primary histology & & & & 0.15 \\
\hline Appendix & $488(46 \%)$ & $240(47 \%)$ & $121(43 \%)$ & \\
\hline Well-differentiated & $253(52 \%)$ & $135(71 \%)$ & $55(29 \%)$ & \\
\hline Moderately differentiated & $156(32 \%)$ & $68(64 \%)$ & $39(36 \%)$ & \\
\hline Poorly differentiated & $77(16 \%)$ & $37(59 \%)$ & $26(41 \%)$ & \\
\hline Colorectal & $309(29 \%)$ & $141(27 \%)$ & $91(32 \%)$ & \\
\hline Peritoneal mesothelioma & $113(11 \%)$ & $59(11 \%)$ & $22(8 \%)$ & \\
\hline Ovarian & $57(5 \%)$ & $25(5 \%)$ & $21(8 \%)$ & \\
\hline Other & $101(9 \%)$ & $51(10 \%)$ & $25(9 \%)$ & \\
\hline ECOG status & & & & 0.20 \\
\hline 0 & $367(34 \%)$ & $190(37 \%)$ & $88(32 \%)$ & \\
\hline 1 & $683(64 \%)$ & $320(62 \%)$ & $186(66 \%)$ & \\
\hline$\geq 2$ & $18(2 \%)$ & $6(1 \%)$ & $6(2 \%)$ & \\
\hline Preoperative chemotherapy & $642(60 \%)$ & $301(58 \%)$ & $195(70 \%)$ & 0.002 \\
\hline Number of cycles & $6(5-10)$ & $6(5-9)$ & $6(5-10)$ & 0.49 \\
\hline \multicolumn{5}{|l|}{ Operative factors } \\
\hline PSS & & & & 0.85 \\
\hline 0 & $62(15 \%)$ & $77(15 \%)$ & $37(13 \%)$ & \\
\hline 1 & $314(30 \%)$ & $147(29 \%)$ & $87(32 \%)$ & \\
\hline 2 & $356(34 \%)$ & $171(34 \%)$ & $91(33 \%)$ & \\
\hline 3 & $220(21 \%)$ & $114(22 \%)$ & $61(20 \%)$ & \\
\hline PCI & $14(8-21)$ & $13(8-20)$ & $15(9-23)$ & 0.004 \\
\hline OR time, hours & $8.3(6.8-10.3)$ & $8.1(6.6-10.0)$ & $8.8(7.2-10.9)$ & $<0.001$ \\
\hline Estimated blood loss, $\mathrm{mL}$ & $500(250-900)$ & $500(250-800)$ & $500(300-900)$ & 0.31 \\
\hline Intraoperative transfusion & $231(22 \%)$ & $103(20 \%)$ & $69(25 \%)$ & 0.13 \\
\hline Number of visceral resections & $2(1-4)$ & $2(1-4)$ & $3(2-4)$ & $<0.001$ \\
\hline Number of anastomoses & $1(0-2)$ & $1(0-2)$ & $1(1-2)$ & 0.001 \\
\hline CC score & & & & 0.21 \\
\hline 0 & $786(74 \%)$ & $389(75 \%)$ & $195(70 \%)$ & \\
\hline 1 & $240(22 \%)$ & $107(21 \%)$ & $73(26 \%)$ & \\
\hline $2+$ & $39(4 \%)$ & $20(4 \%)$ & $11(4 \%)$ & \\
\hline
\end{tabular}


TABLE 2 continued

\begin{tabular}{|c|c|c|c|c|}
\hline \multirow[t]{2}{*}{ Variable } & \multirow[t]{2}{*}{ Entire cohort $[n=1068]$} & \multicolumn{3}{|c|}{ Univariate analysis in the derivation set $[n=796]$} \\
\hline & & No readmission $[n=516]$ & Readmitted $[n=280]$ & $p$-Value \\
\hline \multicolumn{5}{|l|}{ Hospitalization factors } \\
\hline Length of stay, days & $12(9-17)$ & $11(9-15)$ & $13(10-20)$ & $<0.001$ \\
\hline In-hospital complication & $819(76.7 \%)$ & $380(74 \%)$ & $231(83 \%)$ & 0.005 \\
\hline CCI score & $22.6(8.7-36.2)$ & $20.9(0-33.5)$ & $29.6(20.9-45.6)$ & $<0.001$ \\
\hline Major complication & $247(23 \%)$ & $100(19 \%)$ & $92(33 \%)$ & $<0.001$ \\
\hline Leak diagnosed in hospital & $103(10 \%)$ & $37(7 \%)$ & $37(13 \%)$ & 0.006 \\
\hline VTE event in hospital & $66(6 \%)$ & $22(4 \%)$ & $27(10 \%)$ & 0.003 \\
\hline Postoperative transfusion & $503(47 \%)$ & $218(42 \%)$ & $164(59 \%)$ & $<0.001$ \\
\hline Percutaneous drain & $208(20 \%)$ & $87(17 \%)$ & $74(26 \%)$ & 0.001 \\
\hline In-hospital TPN & $190(18 \%)$ & $82(16 \%)$ & $63(23 \%)$ & 0.02 \\
\hline Return to ICU & $114(11 \%)$ & $39(8 \%)$ & $42(15 \%)$ & 0.001 \\
\hline Return to OR & $91(8.5 \%)$ & $27(5 \%)$ & $37(13 \%)$ & $<0.001$ \\
\hline \multicolumn{5}{|l|}{ Discharge factors } \\
\hline Discharge with ostomy & $406(38.0 \%)$ & $165(32 \%)$ & $135(48 \%)$ & $<0.001$ \\
\hline Ileostomy & $370(91.1 \%)$ & $153(93 \%)$ & $122(90 \%)$ & \\
\hline Colostomy & $36(8.9 \%)$ & $12(7 \%)$ & $13(10 \%)$ & \\
\hline Day-of-discharge hemoglobin & $8.9(8.3-9.9)$ & $9.1(8.3-10.0)$ & $8.8(8.1-9.8)$ & 0.005 \\
\hline Day-of-discharge white blood cell count & $7.3(5.0-10.4)$ & $7.0(4.9-9.9)$ & $7.9(5.4-11.0)$ & 0.001 \\
\hline Day-of-discharge sodium & $136(134-138)$ & $136(134-138)$ & $135(133-138)$ & 0.003 \\
\hline Discharge on antibiotics & $197(18.5 \%)$ & $80(16 \%)$ & $68(24 \%)$ & 0.003 \\
\hline Discharge on therapeutic anticoagulation & $121(11.3 \%)$ & $50(10 \%)$ & $38(14 \%)$ & 0.10 \\
\hline Discharge on regular diet & $991(92.8 \%)$ & $489(95 \%)$ & $250(89 \%)$ & 0.005 \\
\hline Discharge with TPN & $90(8.4 \%)$ & $36(7 \%)$ & $35(13 \%)$ & 0.01 \\
\hline Number of discharge medications & $8(5-10)$ & $7(5-10)$ & $8(6-12)$ & $<0.001$ \\
\hline Discharge to a skilled nursing facility & $86(8.1 \%)$ & $24(5 \%)$ & $36(13 \%)$ & $<0.001$ \\
\hline
\end{tabular}

Data are displayed as mean (SD), median (IQR), or count (\%)

$A A-C C I$ age-adjusted Charlson-Deyo Comorbidity Index, ASA American Society of Anesthesiologists, PSS prior surgical score, PCI Peritoneal Cancer Index, $C C$ completeness of cytoreduction, $C C I$ Comprehensive Complication Index, BMI body mass index, IQR interquartile range, $C R S$ cytoreductive surgery, HIPEC hyperthermic intraperitoneal chemotherapy, ECOG Eastern Cooperative Oncology Group, OR operating room, $V T E$ venous thromboembolism, TPN total parenteral nutrition, $I C U$ intensive care unit, $S D$ standard deviation

TABLE 3 Derivation of model and score

\begin{tabular}{llllll}
\hline Variable & OR & $95 \%$ CI & $\beta$ coefficient & $p$-Value & Point value \\
\hline Stoma & 1.41 & $1.02-1.96$ & 0.345 & 0.04 & 1 \\
PCI $\geq 15$ & 1.43 & $1.04-1.96$ & 0.358 & 0.03 & 1 \\
Sodium $<135 \mathrm{mEq} / \mathrm{L}^{\mathrm{a}}$ & 1.51 & $1.10-2.08$ & 0.412 & 0.01 & 1 \\
Major complication & 1.54 & $1.07-2.20$ & 0.430 & 0.02 & 1 \\
Preoperative chemotherapy & 1.63 & $1.18-2.26$ & 0.488 & 0.00 & 1 \\
Hemoglobin $<8 \mathrm{~g} / \mathrm{dL}^{\mathrm{a}}$ & 1.78 & $1.18-2.69$ & 0.579 & 0.01 & 2 \\
Discharge to SNF & 2.63 & $1.48-4.67$ & 0.967 & 0.00 & 3 \\
& & & & Total & 10 \\
\hline
\end{tabular}

${ }^{a}$ Laboratory values taken on the day of discharge

PCI Peritoneal Cancer Index, SNF skilled nursing facility, OR odds ratio, CI confidence interval 


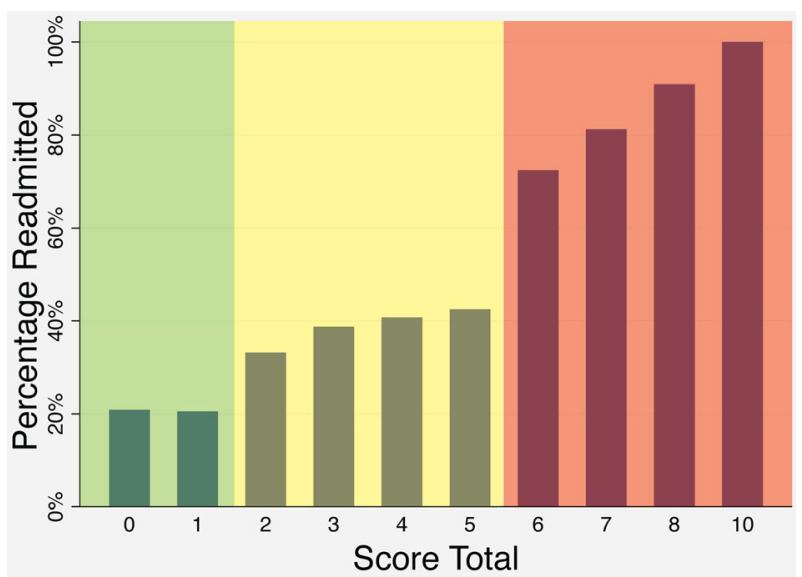

FIG. 2 Percentage of patients readmitted in the derivation cohort, by score total. Patients were divided into low (0-2), moderate (2-5), and high-risk (6+) cohorts, based on the rate of 90-day readmission

\section{DISCUSSION}

In this study of 1068 encounters for CRS/HIPEC, we developed and internally validated a risk prediction tool for 90-day readmission following surgery. We found that at the time of discharge, seven factors easily and accurately stratified risk for readmission: stoma creation, PCI score, hyponatremia, major complications, preoperative chemotherapy, anemia, and discharge to a nursing facility. Our tool demonstrated good calibration and fair discrimination, suggesting that even within a high-risk population, it is possible to stratify risk for readmission to target a higher risk cohort for intervention.

These parameters are objective and are readily available at the time of discharge. Using this tool, $61 \%$ of our total CRS/HIPEC population was classified as moderate risk for readmission (scores of 2-5), with a predicted rate of readmission of $38 \%$ and an observed rate of $38 \%$. An additional $8 \%$ were classified as high risk for readmission (scores 6+), with a predicted rate of readmission of $69 \%$ and an observed rate of readmission of $78 \%$. A similar performance in predicting overall readmissions and surgical quality readmissions suggests this score could be clinically applicable in targeting preventable readmissions. ${ }^{11}$

At present, the discharge process at our institution is not protocolized. All CRS/HIPEC patients receive a 30-day supply of prophylactic anticoagulation and routine home health care; however, time to postoperative follow-up, telephone and telemedicine check-ins, and other services are variable. Ideally, patients would receive targeted follow-up and specific interventions tailored to their needs and relative risk for readmission. Identifying which patients are most at risk and likely to benefit from intervention is a crucial first step in improving readmission rates.

Drivers of readmission following surgery are complex and varied. As a consequence, published interventions to prevent readmission are diverse. In a systematic review examining readmissions for medical and surgical patients, Leppin and colleagues found an overall reduction in the risk of readmission of $18 \%$ associated with readmission reduction programs across the 42 examined studies. ${ }^{18}$ The authors suggest that multimodal interventions focused on coordinating care and increasing patients' ability to engage in self-care postdischarge were most effective at reducing readmissions. Similarly, Jones et al. examined transitional care interventions after surgery and found that coordinated discharge planning, patient education, and protocolized follow-up reduced readmissions. ${ }^{19}$ Evaluated programs demonstrated reductions in readmissions ranging from 8 to $24 \%$. In both reviews, the authors stressed that successful programs offered comprehensive predischarge assessment of needs, together with coordinated discharge and tailored postdischarge follow-up.

We propose to use our score to develop and implement an evidence-driven quality improvement program to address readmission following CRS/HIPEC at our institution. Theoretical application of our score, utilizing a cut-off

TABLE 4 Observed versus predicted readmission

\begin{tabular}{lcccc}
\hline Points & Risk category & $\begin{array}{l}\text { Number of } \\
\text { patients }(\%)\end{array}$ & $\begin{array}{l}\text { Observed rate of } \\
\text { readmission }(\%)\end{array}$ & $\begin{array}{l}\text { Predicted rate of } \\
\text { readmission }(\%)\end{array}$ \\
\hline $\begin{array}{l}\text { Derivation cohort }[n=796] \\
0-1\end{array}$ & Low & & & 20.7 \\
$2-5$ & Moderate & $247(31.0)$ & 37.4 & 38.1 \\
$6+$ & High & $492(61.8)$ & 79.0 & 69.3 \\
Validation cohort $[n=272]$ & $57(7.2)$ & & 21.4 \\
$0-1$ & Low & & 17.6 & 38.1 \\
$2-5$ & Moderate & $91(33.4)$ & 41.0 & 69.3 \\
$6+$ & High & $156(57.4)$ & 76.0 & \\
\hline
\end{tabular}


of 2 and above to target patients for readmission intervention, would target $68 \%$ of our cohort and $82 \%$ of all readmitted patients, with a sensitivity of $82 \%$, specificity of $39 \%$, and AUC of 0.61 (electronic supplementary Table 6). We plan to target moderate- and high-risk patients through tailored advanced discharge planning addressing nutritional needs, fluid status, pain management, and wound/stoma care, as well as graduated advance practice provider telemedicine and in-person follow-up based on risk score.

Our overall readmission rate of $35.5 \%$ is consistent with prior reports, however notably somewhat higher than other high-volume centers. ${ }^{1,3}$ As other CRS/HIPEC readmission studies have demonstrated, we found complications were predictive of readmission. ${ }^{12-14}$ Our model derivation included models with both composite measures of complications (CCI total, major complications) and individual complications (VTE, leak, reoperation). Ultimately, in multivariate analysis, the presence of a major complication remained a strong predictor of readmission.

Several measures of operative complexity and specific procedures were examined for association with readmissions. While many measures, including operative length, blood loss, number of resections, and number of anastomoses, were significant on univariate analysis, stoma creation, which included both ileostomy and colostomy creation, remained significant on multivariate analysis. Ostomies are recognized risk factors for readmission following CRS/HIPEC and general colorectal surgery. ${ }^{3,13,20}$ Stoma creation may be a surrogate marker of more complex surgery. Additionally, stoma creation, particularly among elderly patients, places patients at higher risk for fluid and electrolyte issues, a common indication for readmission in our cohort. ${ }^{20}$

In our final model, higher PCI score was associated with readmission. This is notable in that previous studies have suggested PCI scores were not independently associated with readmission. In their examination of 223 patients undergoing CRS/HIPEC, Dreznik and colleagues ${ }^{14}$ found no difference in PCI score between patients with and without readmission (mean PCI 12.6 vs. 12.4, $p=0.53$ ). Lee and colleagues ${ }^{3}$ conducted a multi-institutional review of readmissions at 12 high-volume CRS/HIPEC centers, and, in their study of 2017 cases, they examined preoperative and operative predictors of readmission. There was a trend toward higher PCI scores in the readmitted cohort (15 vs. $13, p=0.07$ ); however, on multivariate analysis, PCI scores above 20 were not associated with readmission ( $p=0.21$ ). Our population had overall higher PCI scores, potentially signifying higher preoperative disease burden and accounting for differences between this and prior studies. Additionally, our model included postoperative and day-of-discharge variables not included in the analysis by Lee et al., which may underlie model differences.
Pre-CRS/HIPEC chemotherapy emerged as a significant predictor of readmission. In their examination of readmissions, Lee et al. found neoadjuvant chemotherapy to be associated with readmission on univariate analysis $(p<0.01)$, however in their ultimate multivariate model, it did not remain a significant predictor $(p=0.59){ }^{3}$ Notably, preoperative chemotherapy rates were significantly higher in our cohort $(60 \%)$, with relatively high preoperative chemotherapy burden (six cycles, IQR 5-10). This higher burden and duration of chemotherapy may account for differences in significance.

Day-of-discharge laboratory studies, hemoglobin and sodium, were also significant predictors of readmission; both have previously been found to be significant predictors of readmission. Merkow et al. examined nearly 500,000 patients undergoing surgery in the US and found that complications due to bleeding or anemia were the third most common cause of readmission. ${ }^{21}$ In their analysis of readmissions following CRS/HIPEC, Martin et al. found perioperative transfusion was associated with readmission at 30 days. $^{12}$ In our analysis, while perioperative transfusion was predictive of readmission by univariate analysis, only anemia on the day of discharge remained significant on multivariate analysis. Similarly, hyponatremia was found to be significantly associated with readmission in our final analysis. Hyponatremia features in several common risk prediction models, such as the HOSPITAL and MELD-Na scores, and has been independently associated with increased complications and mortality in cardiac surgery patients. ${ }^{10,22,23}$ Underlying mechanisms for this association remain unclear, however hyponatremia has been identified as a marker of systemic inflammation and associated infection. ${ }^{24}$ Given the high rates of infection among CRS/HIPEC readmissions, these patients may be showing early signs at the time of discharge. Alternatively, these laboratory values may function more as general markers of prognosis and comorbidities, with sodium reflecting protein malnutrition and anemia signifying marrow suppression due to preoperative or intraoperative chemotherapy.

Finally, discharge to a skilled nursing facility emerged as the highest weighted factor within our model despite not being associated with readmission in previous studies. ${ }^{3,13}$ Notably, a significantly higher portion of our population, i.e. $8 \%$ of our cohort, was discharged to a nursing facility following index hospitalization. Among discharges examined by Lee et al. and Kelly et al., $2.7 \%$ and $3.1 \%$ of patients were discharged to a nursing home, respectively. It is possible that in our model, discharge to a nursing facility stands in for potentially unmeasured differences in physical status or frailty, accounting for differences in significance between studies. 
This study has several notable limitations. Although we examined readmissions out to 90 days, including both index and non-index hospitalizations, it is possible that additional patients were readmitted to non-index facilities without our knowledge. However, our similar readmission rates with those previously reported suggested that the portion of missed readmissions is likely small, or at least on par with previous studies. ${ }^{3}$ Therefore, we do not feel there is sufficient evidence to suggest this portion of missed readmissions would significantly alter our findings. Additionally, although we excluded in-hospital mortalities and discharges to hospice, there is the potential that patients died after discharge without readmission. We examined 90-day survival after discharge within our cohort, with mortalities verified by the National Death Index; 26 patients $(2.4 \%)$ died within 90 days of discharge. All of these patients were readmitted first, suggesting that the potential influence of missed mortalities would be low. Finally, this study only performed an internal validation of our model and is drawn from the experience of a single, high-volume center. Limited numbers of patients with scores above 8 impairs calibration and estimation. Both prospective and external validation is necessary to further refine our stratification and improve our risk score.

This study represents a novel risk prediction tool in a high-risk population. In similar prediction models, diagnosis with malignancy or disseminated cancer is heavily weighted towards readmission, making such tools less useful when addressing a surgical oncology population. In this study, we show that even among patients with baseline high risk for readmission, we can stratify risk to target future efforts to the most vulnerable cohort in an effort to improve patient outcomes.

\section{CONCLUSIONS}

In this analysis, we derive and validate a practical prediction model to assess risk for 90-day readmission in patients undergoing CRS/HIPEC. To our knowledge, this represents the first risk prediction tool specifically generated for this complex population. We propose to use this score to target moderate and high-risk patients for enhanced discharge planning and intensive postdischarge monitoring and follow-up.

ACKNOWLEDGMENT Research reported in this publication was supported by the National Cancer Institute of the National Institutes of Health under award number T32CA113263. The content is solely the responsibility of the authors and does not necessarily represent the official views of the National Institutes of Health.

OPEN ACCESS This article is licensed under a Creative Commons Attribution 4.0 International License, which permits use, sharing, adaptation, distribution and reproduction in any medium or format, as long as you give appropriate credit to the original author(s) and the source, provide a link to the Creative Commons licence, and indicate if changes were made. The images or other third party material in this article are included in the article's Creative Commons licence, unless indicated otherwise in a credit line to the material. If material is not included in the article's Creative Commons licence and your intended use is not permitted by statutory regulation or exceeds the permitted use, you will need to obtain permission directly from the copyright holder. To view a copy of this licence, visit http://creativecommons. org/licenses/by/4.0/.

\section{REFERENCES}

1. Choudry MHA, Shuai Y, Jones HL, et al. Postoperative complications independently predict cancer-related survival in peritoneal malignancies. Ann Surg Oncol. 2018;25(13):3950-9. h ttps://doi.org/10.1245/s10434-018-6823-9.

2. Lee L, Alie-Cusson F, Dube P, Sideris L. Postoperative complications affect long-term outcomes after cytoreductive surgery and hyperthermic intraperitoneal chemotherapy for colorectal peritoneal carcinomatosis. J Surg Oncol. 2017;116(2):236-43. h ttps://doi.org/10.1002/jso.24632.

3. Lee TC, Wima K, Sussman JJ, et al. Readmissions after cytoreductive surgery and hyperthermic intraperitoneal chemotherapy: a US HIPEC collaborative study. $J$ Gastrointest Surg. 2020;24(1):165-76. https://doi.org/10.1007/s11605-019-04463-y.

4. Zafar SN, Shah AA, Channa H, Raoof M, Wilson L, Wasif N. Comparison of rates and outcomes of readmission to index vs nonindex hospitals after major cancer surgery. JAMA Surg. 2018;153(8):719-27. https://doi.org/10.1001/jamasurg.2018. 0380.

5. Zheng C, Habermann EB, Shara NM, et al. Fragmentation of care after surgical discharge: non-index readmission after major cancer surgery. J Am Coll Surg. 2016;222(5):780-789.e2. https://doi. org/10.1016/j.jamcollsurg.2016.01.052.

6. Schneider MA, Eshmuminov D, Lehmann K. Major postoperative complications are a risk factor for impaired survival after CRS/HIPEC. Ann Surg Oncol. 2017;24(8):2224-32. https://doi. org/10.1245/s10434-017-5821-7.

7. Tevis SE, Weber SM, Kent KC, Kennedy GD. Nomogram to predict postoperative readmission in patients who undergo general surgery. JAMA Surg. 2015;150(6):505-10. https://doi.org/10. 1001/jamasurg.2014.4043.

8. Kassin MT, Owen RM, Perez SD, et al. Risk factors for 30-day hospital readmission among general surgery patients. $J$ Am Coll Surg. 2012;215(3):322-30. https://doi.org/10.1016/j.jamcollsurg. 2012.05.024.

9. McIlvennan CK, Eapen ZJ, Allen LA. Hospital readmissions reduction program. Circulation. 2015;131(20):1796-803. http s://doi.org/10.1161/circulationaha.114.010270.

10. Donze J, Aujesky D, Williams D, Schnipper JL. Potentially avoidable 30-day hospital readmissions in medical patients: derivation and validation of a prediction model. JAMA Intern Med. 2013;173(8):632-8. https://doi.org/10.1001/jamainternmed. 2013.3023.

11. Mull HJ, Graham LA, Morris MS, et al. Association of postoperative readmissions with surgical quality using a Delphi consensus process to identify relevant diagnosis codes. JAMA Surg. 2018;153(8):728-37. https://doi.org/10.1001/jamasurg.201 8.0592 .

12. Martin AS, Abbott DE, Hanseman D, et al. Factors associated with readmission after cytoreductive surgery and hyperthermic intraperitoneal chemotherapy for peritoneal carcinomatosis. Ann 
Surg Oncol. 2016;23(6):1941-7. https://doi.org/10.1245/s10434016-5109-3.

13. Kelly KJ, Cajas L, Baumgartner JM, Lowy AM. Factors associated with 60-day readmission following cytoreduction and hyperthermic intraperitoneal chemotherapy. Ann Surg Oncol. 2018;25(1):91-7. https://doi.org/10.1245/s10434-017-6108-8.

14. Dreznik Y, Hoffman A, Hamburger T, et al. Hospital readmission rates and risk factors for readmission following cytoreductive surgery (CRS) and hyperthermic intraperitoneal chemotherapy (HIPEC) for peritoneal surface malignancies. Surgeon. 2018;16(5):278-82. https://doi.org/10.1016/j.surge.2018.01.001.

15. White IR, Royston P, Wood AM. Multiple imputation using chained equations: issues and guidance for practice. Stat Med. 2011;30(4):377-99. https://doi.org/10.1002/sim.4067.

16. Prince Nelson SL, Ramakrishnan V, Nietert PJ, Kamen DL, Ramos PS, Wolf BJ. An evaluation of common methods for dichotomization of continuous variables to discriminate disease status. Commun Stat Theory Methods. 2017;46(21):10823-34. h ttps://doi.org/10.1080/03610926.2016.1248783.

17. Moons KGM, Harrell FE, Steyerberg EW. Should scoring rules be based on odds ratios or regression coefficients? J Clin Epidemiol. 2002;55(10):1054-5. https://doi.org/10.1016/s08954356(02)00453-5.

18. Leppin AL, Gionfriddo MR, Kessler M, et al. Preventing 30-day hospital readmissions: a systematic review and meta-analysis of randomized trials. JAMA Intern Med. 2014;174(7):1095-107. h ttps://doi.org/10.1001/jamainternmed.2014.1608.

19. Jones CE, Hollis RH, Wahl TS, et al. Transitional care interventions and hospital readmissions in surgical populations: a systematic review. Am J Surg. 2016;212(2):327-35. https://doi. org/10.1016/j.amjsurg.2016.04.004.

20. Chen SY, Stem M, Cerullo M, et al. Predicting the risk of readmission from dehydration after ileostomy formation: the dehydration readmission after ileostomy prediction score. Dis Colon Rectum. 2018;61(12):1410-7. https://doi.org/10.1097/dcr. 0000000000001217.

21. Merkow RP, Ju MH, Chung JW, et al. Underlying reasons associated with hospital readmission following surgery in the United States. JAMA. 2015;313(5):483-95. https://doi.org/10.10 01/jama.2014.18614.

22. Biggins SW, Kim WR, Terrault NA, et al. Evidence-based incorporation of serum sodium concentration into MELD. Gastroenterology. 2006;130(6):1652-60. https://doi.org/10.1053/j.ga stro.2006.02.010.

23. Crestanello JA, Phillips G, Firstenberg MS, et al. Postoperative hyponatremia predicts an increase in mortality and in-hospital complications after cardiac surgery. $J$ Am Coll Surg. 2013;216(6):1135-43. https://doi.org/10.1016/j.jamcollsurg.2013 .02 .010 .

24. Swart RM, Hoorn EJ, Betjes MG, Zietse R. Hyponatremia and inflammation: the emerging role of interleukin-6 in osmoregulation. Nephron Physiol. 2011;118(2):45-51. https://doi.org/10. 1159/000322238.

Publisher's Note Springer Nature remains neutral with regard to jurisdictional claims in published maps and institutional affiliations. 